\title{
Skills Training in Prosthodontics - A Report
}

\section{Pradeep Dathan ${ }^{1}$, K Chandrasekharan Nair ${ }^{2 *}$, Bhimalingesvara Rao $^{3}$ and Mohan Kumar $\mathbf{T}^{4}$}

${ }^{1}$ Professor and Head of the Department of Prosthodontics, Sri Sankara Dental

College, Akathumuri, Thiruvavananthapuram, Kerala, India

${ }^{2}$ Professor Emeritus, Department of Prosthodontics, Sri Sankara Dental College,

Akathumuri, Thiruvananthapuram, Kerala, India

${ }^{3}$ Professor of Prosthodontics, Vishnu Dental College, Bhimavaram, Andhra Pradesh, India

${ }^{4}$ Professor of Prosthodontics, College of Dental Sciences and Hospital, Rau, Indore, India

*Corresponding Author: K Chandrasekharan Nair, Professor Emeritus,

Department of Prosthodontics, Sri Sankara Dental College, Akathumuri,

Thiruvananthapuram, Kerala, India.
Received: September 01, 2021

Published: September 27, 2021

(C) All rights are reserved by

K Chandrasekharan Nair., et al.

\section{Abstract}

Skills training in Prosthodontics, requires time, teachers and repetitions by the students. When proficiency is achieved, performance of skill becomes automatic. Pandemic has deprived the students from attending practical and clinical classes. Professionally it is a great loss for the present day students. Basics of skill training, effect of the pandemic on skill training, compensatory mechanisms to be adopted and competency based training and assessment are discussed in this report.

Keywords: Skills Training; Repetition of Skills; Proficiency; Evolution of Skills Training; Pandemic

\section{Introduction}

Educational training in dentistry has always focused on three foundational areas viz. knowledge, skills and attitude. Knowledge sector always gained prime focus and educational research centered round making clear objectives, teaching learning methods and objective assessment practices. Skills training in dentistry, especially in prosthodontics was much evolved from the initial phases of dental education. In fact skills training superseded knowledge and attitude in dentistry. This article focuses on the evolution of skills training in Prosthodontics in the Indian context.

History of skills training in prosthodontics

Skills training in Prosthodontics have a long history which can be traced back to the use of extracted teeth. This practice we cannot claim to have phased out completely in India. Later Oswald Ferguson designed the phantom head simulator in the year 1894.
Modern phantom heads include dental hand pieces, water sprays etc. Extracted teeth were replaced with plastic teeth in due course of time. The tooth preparation in phantom head is an irreversible experiment. Though effective, it is expensive and not environment friendly. Phantom heads were followed by the dental simulator which could simulate realistic clinical conditions via Virtual Reality (VR) and force feedback. Simulators make training reversible, repeatable, and environment friendly [1].

Evolution of prosthodontic skills training

A new entrant to the BDS course is exposed to a series of practical exercises related to complete denture fabrication - making impressions, casts, temporary bases, wax occlusion rims, familiarizing with Gysi's fixed path articulator, mounting the casts in the articulator, arranging teeth, wax carving, processing the dentures in acrylic, finishing and polishing the dentures and denture repairing. 
Five repetitions of the processing was a minimum requirement. Minimum 8 to 10 hours of preclinical training was given in one week. In the second year BDS, the preclinical training consisted of exercises related to removable partial denture fabrication belonging to Kennedy class 1 to 4 . Crown and bridge preparations were made on big sized plaster/wax models followed by the preparations on phantom plastic teeth was done in the III and IV BDS. Preclinical training was carried out under supervision of the teachers and repetitions were common so that students excelled in quality.

As years passed by, the importance and duration of preclinical training in prosthodontics got reduced because of the inclusion of too many newer preclinical exercises related to orthodontics, pedodontics, dental implantology etc. The usefulness of preclinical training is not convincingly explained to the students. The teachers themselves were not fully convinced of the importance of preclinical training. Proficiency in a skill is achieved in three stages. The teacher demonstrates the skill and the students observe. Later the students imitate the skill under the supervision of the teacher. In the third stage, the student will be able to perform independently and repeatedly until proficiency is achieved. A proficient student can tackle emergency situations efficiently. The fact to be noticed is that, honing the skill perfectly well is the result of repeatedly doing it.

Jerry Uelsmann, a professor of photography in the University of Florida, has narrated an experiment he conducted in the class. He divided the class into two groups. The first group was asked to take as many photographs as possible whereas the second group was asked to produce the best quality photograph. The former group concentrated on the quantity whereas the latter group spent much of their time in contemplating on the quality of photograph they have to produce. The skills of the first group were honed very well and produced many good photographs. The latter group produced hardly one photograph and that too of mediocre quality. The importance of repetition in gaining proficiency is evident in this incident [2].

\section{Repeatedly doing the skill makes it a habit}

By repeatedly doing a skill, that behavior progressively becomes automatic. By repetition of an activity, the structure of the brain becomes efficient in that activity. By repeating an activity, it becomes a habit and by repeating a habit, clear physical changes happen in the brain. By repetition, the concerned cell to cell signaling improves and neural connections tighten. Neuropsychologist Donald
Hebb has stated that "Neurons that wire together fire together" (Hebb's axiom reminds us that every experience, thought, feeling, and physical sensation triggers thousands of neurons, which form a neural network. When you repeat an experience over and over, the brain learns to trigger the same neurons each time). Each time an action is repeated, particular neural circuit associated with that is activated. Simply by making repetitions, encodes a new habit and the activity becomes automatic. That is why the teachers have fixed up a quota for the practical and clinical activities. Value of this, each professional will experience, later in their practice [2] (Figure 1-3).

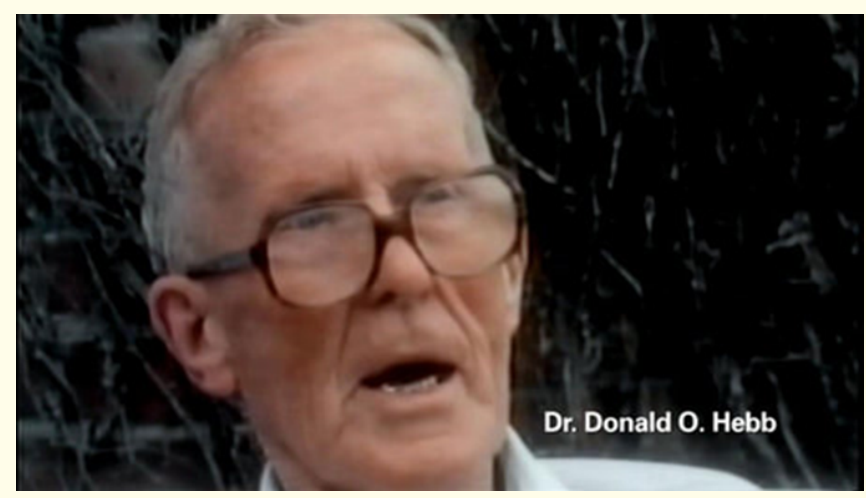

Figure 1: Dr. Donald O Hebb.

Source: https://www.theeducatedbrain.com/neuroplasticity.html.

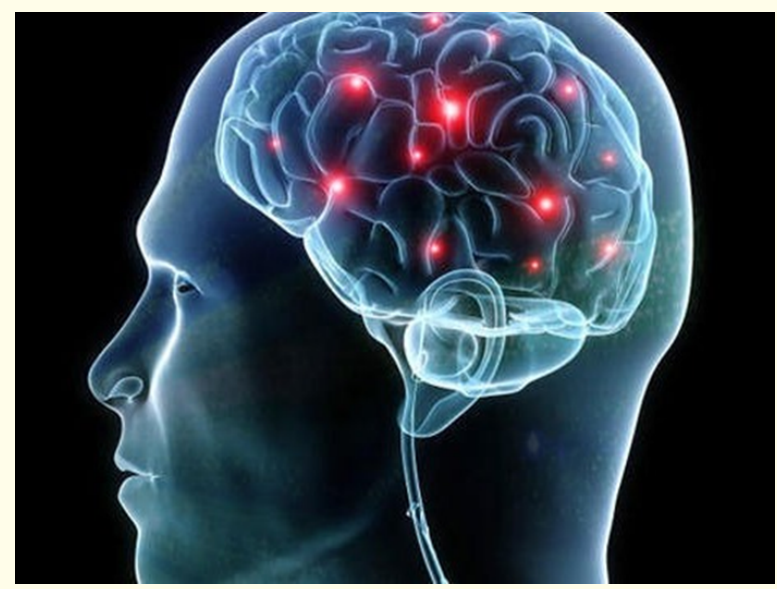

Figure 2: Neurons that fire together wire together.

Source: https://www.supercamp.com/what-does-neurons-that-firetogether-wire-together-mean/. 
The more you do something, the more your brain responds to support that activity.

Figure 3: Hebb's statement on skills' training.

\section{How the pandemic has affected skills training}

In the month of April, 2019 the Covid-19 pandemic had its onslaught on Indian professional education. Dental colleges were closed because the country has gone to a complete lock down. Clinical and preclinical sessions were almost completely cancelled. Only online lecture classes were conducted. A year has passed without giving students a chance to attend even a singular clinical session. No effective compensatory mechanism was thought of. Practical examinations were also conducted online with the examiner available in a remote place. Postgraduate examinations were also conducted online much to the advantage of the students who were not keen to exert for the examination. Universities have also taken a lenient view on the assessment system. The end result will be evident after a few years; by that time irreparable damage might happen to the professional skills and competencies. What we have to think of at present is about the remedial measures we have to implement in the future. Professional advancement programmes have to be planned for all the undergraduate and postgraduate professionals and if necessary make such programmes mandatory for the renewal of yearly registration.

\section{Competency based assessment}

Alkhodary., et al. have compared traditional way of assessment and Competency Based Assessment (CBA) and found that students could get better scoring with competency based assessment. They have given examples of clinical examination in prosthodontics.
While assessing the skill of jaw relation registration, in the traditional way the teacher would make a global assessment and the student will not be able to understand where he has erred. In the competency based assessment, competencies are listed with gradation norms as follows:

1. Knowledge related to jaw relation:
a. All clinical steps understood (excellent).
b. Some steps understood (Good).
c. No knowledge on clinical steps (Fail).

2. Orientation of occlusal plane:
a. Parallel to inter pupillary and ala tragal lines (excellent).
b. Parallelism achieved with guidance (Good).
c. Occlusal plane needed realignment (Fail).

3. Vertical dimension:
a. VDO less than VDR by $4 \mathrm{~mm}$ (Excellent).
b. VDO less than VDR by $1 \mathrm{~mm}$ (Good).
c. Low/High VD needed remaking (Fail).

4. Horizontal jaw relation:

a. Retruded unstrained position (Excellent).
b. Retruded position, even contact was not present and realigned (Good).

c. Wrong centric (Fail).

5. Lip support/shade selection:

a. Proper support/proper shade (Excellent).

b. Not adequately supported/shade not appropriate (Good).

c. Lip dished in/shade not selected (Fail).

The marks for each step can be assigned by the teachers, colleges, universities or by the councils.

CBA is depended exclusively on the performance of the student and his achievement can be rated by him and possibly can be compared with other students. The factors that are considered for 
assessment and the method of assigning marks will be available for the student beforehand during the training session. If the traditional method is teacher centered, the CBA is student centered. In the post pandemic period, skills training and its assessment can be modified to CBA as an effective compensatory measure and for objective consolidation of our training system [3].

\section{Conclusion}

Skills' training in Prosthodontics is an essential component of the BDS and MDS programme. Students are trained through elaborate preclinical and clinical experiments, which require time and teachers' instruction. Many experiments have to be repeated many times to gain proficiency so that the students will be able to perform with ease and automatism in their professional life.

\section{Bibliography}

1. Yaning Li., et al. "The Current Situation and Future Prospects of Simulators in Dental Education". Journal of Medical Internet Research 23.4 (2021): e23635.

2. James Clear. "Atomic Habits". Penguin Random House, UK (2018): 141-147.

3. Alkhodary MA., et al. "Competency-based education in undergraduate clinical prosthodontics: A paradigm shift in training". Competency-Based Education 5 (2020): e01220.

\section{Volume 5 Issue 10 october 2021}

(C) All rights are reserved by $\mathrm{K}$ Chandrasekharan Nair., et al. 